\title{
REFINEMENTS OF JENSEN'S INEQUALITY
}

\author{
I. BRNETIĆ, C. E. M. PEARCE AND J. PEČARIĆ
}

Abstract. Some new refinements are presented for Jensen's inequality. These strengthen several results obtained in the recent literature.

\section{Introduction}

A central tool in analysis is Hadamard's inequality

$$
f\left(\frac{a+b}{2}\right) \leq \frac{1}{b-a} \int_{a}^{b} f(x) d x \leq \frac{f(a)+f(b)}{2}
$$

for convex functions. Recently some improvements for this have been found by Yang and Wang [3]. In particular they established the following.

Theorem A. Let $f:[a, b] \rightarrow \mathbf{R}$ be a convex function and $\alpha_{i} \in(0,1)(i=1, \ldots, n)$ with $\sum_{i=1}^{n} a_{i}=1$. Then

$$
\begin{aligned}
f\left(\frac{a+b}{2}\right) & \leq \frac{1}{(b-a)^{n}} \int_{a}^{b} \cdots \int_{a}^{b} f\left(\sum_{i=1}^{n} a_{i} x_{i}\right) d x_{1} \cdots d x_{n} \\
& \leq \sum_{i=1}^{n} \frac{1-\alpha_{i}}{(n-1)(b-a)^{n-1}} \int_{a}^{b} \cdots \int_{a}^{b}\left(\frac{\sum_{j=1, j \neq i}^{n} \alpha_{j} x_{j}}{1-\alpha_{i}}\right) \times d x_{1} \cdots d x_{i-1} d x_{i+1} \cdots d x_{n} \\
& \leq \frac{1}{b-a} \int_{a}^{b} f(x) d x .
\end{aligned}
$$

They also constructed a convex, increasing function which lies between the two sides of the first inequality in (1).

Theorem B. Let $f:[a, b] \rightarrow \mathbf{R}$ be a convex function and $\alpha_{i} \in(0,1)(i=1, \ldots, n)$ with $\sum_{i=1}^{n} \alpha_{i}=1$. If $K:[0,1] \rightarrow \mathbf{R}$ is a function defined by

$$
K(t)=\frac{1}{(b-a)^{n}} \int_{a}^{b} \cdots \int_{a}^{b} f\left(t \sum_{i=1}^{n} \alpha_{i} x_{i}+(1-t) \frac{a+b}{2}\right) d x_{1} \cdots d x_{n}
$$

Received March 9, 1999; revised April 4, 1999.

1991 Mathematics Subject Classification. Primary 26D15, secondary 26A51.

Key words and phrases. Jensen's inequality, convexity. 
then

(i) $K$ is convex on $[0,1]$,

(ii)

$$
\begin{aligned}
& \min _{t \in[0,1]} K(t)=K(0)=f\left(\frac{a+b}{2}\right) \\
& \max _{t \in[0,1]} K(t)=K(1)=\frac{1}{(b-a)^{n}} \int_{a}^{b} \cdots \int_{a}^{b} f\left(\sum_{i=1}^{n} \alpha_{i} x_{i}\right) d x_{1} \cdots d x_{n},
\end{aligned}
$$

(iii) $K$ is increasing on $[0,1]$.

In this paper we make some analogous improvements to Jensen's inequality. In Section 2 we derive the first of these. An important special case extends Theorem A. A related result provides a further interpolation of our first result. In Section 3 we present a convex function construction analogous to that of Theorem B. Finally in Section 4 we give a second improvement of Jensen's inequality. This extends a result of Yang and Wu [4].

We suppose without further comment the existence of all the integrals in our discussion. We also suppose $n \geq 2$ throughout, as the statements for $n=1$ are either trivial or void.

\section{First Refinement}

Theorem 1. Suppose $I$ is a real interval. Let $f: I \rightarrow \mathbf{R}$ be a convex function, $g:[a, b] \rightarrow I$ a real function and $w:[a, b] \rightarrow \mathbf{R}$ a positive function. Let $\alpha_{i} \in(0,1)$ $(i=1, \ldots, n)$ with $\sum_{i=1}^{n} \alpha_{i}=1$ and $\bar{w}=\int_{a}^{b} w(x) d x$. Further, let

$F_{k, n}=\frac{1}{\left(\begin{array}{l}n-1 \\ k-1\end{array}\right) \bar{w}^{k}} \sum_{1 \leq i_{1}<\cdots<i_{k} \leq n} \sum_{r=1}^{k} \alpha_{i_{r}} \int_{a}^{b} \cdots \int_{a}^{b}\left[\prod_{s=1}^{k} w\left(x_{i_{s}}\right)\right] f\left(\frac{\sum_{j=1}^{k} \alpha_{i_{j}} g\left(x_{i_{j}}\right)}{\sum_{j=1}^{k} \alpha_{i_{j}}}\right) d_{x_{i_{1}}} \cdots d x_{i_{k}}$.

Then

$$
f\left(\frac{1}{\bar{w}} \int_{a}^{b} w(x) g(x) d x\right) \leq F_{n, n} \leq \cdots \leq F_{k+1, n} \leq F_{k, n} \leq \cdots \leq F_{1, n}=\frac{1}{\bar{w}} \int_{a}^{b} w(x) f(g(x)) d x .
$$

Proof. Using Jensen's inequality, we obtain

$$
\begin{aligned}
f\left(\frac{1}{\bar{w}} \int_{a}^{b} w(x) g(x) d x\right) & =f\left(\frac{1}{\bar{w}^{n}} \int_{a}^{b} \cdots \int_{a}^{b}\left[\prod_{j=1}^{n} w\left(x_{j}\right)\right] \sum_{j=1}^{n} \alpha_{i} g\left(x_{i}\right) d x_{1} \cdots d x_{n}\right) \\
& \leq \frac{1}{\bar{w}} \int_{a}^{b} \cdots \int_{a}^{b}\left[\prod_{j=1}^{n} w\left(x_{j}\right)\right] f\left(\sum_{i=1}^{n} \alpha_{i} g\left(x_{i}\right)\right) d x_{1} \cdots d x_{n}
\end{aligned}
$$


which establishes the first inequality in (2).

For all $k=1, \ldots, n$, define

$$
f_{k, n}\left(x_{1}, \ldots, x_{n}\right)=\frac{1}{\left(\begin{array}{c}
n-1 \\
k-1
\end{array}\right)} \sum_{1 \leq i_{1}<\cdots<i_{k} \leq n} \sum_{r=1}^{k} \alpha_{i_{r}} f\left(\frac{\sum_{j=1}^{k} \alpha_{i_{j}} x_{i_{j}}}{\sum_{j=1}^{k} \alpha_{i_{j}}}\right) .
$$

It has been shown in [2] that

$$
f_{k+1, n}\left(x_{1}, \ldots, x_{n}\right) \leq f_{k, n}\left(x_{1}, \ldots, x_{n}\right)
$$

for all $k=1, \ldots, n-1$ and all real $n$-tuples $\left(x_{1}, \ldots, x_{n}\right)$, so in particular we have

$$
f_{k+1, n}\left(g\left(x_{1}\right), \ldots, g\left(x_{n}\right)\right) \leq f_{k, n}\left(g\left(x_{1}\right), \ldots, g\left(x_{n}\right)\right) .
$$

On multiplying by $\prod_{\ell=1}^{n} w\left(x_{\ell}\right)$ and integrating with respect to $x_{1}, x_{2}, \ldots, x_{n}$, we obtain

$$
\begin{aligned}
& \frac{\bar{w}^{n-k-1}}{\left(\begin{array}{c}
n-1 \\
k
\end{array}\right)} \sum_{1 \leq i_{1}<\cdots<i_{k+1} \leq n} \sum_{r=1}^{k+1} \alpha_{i_{r}} \int_{a}^{b} \cdots \int_{a}^{b}\left[\prod_{s=1}^{k+1} w\left(x_{i_{s}}\right)\right] f\left(\frac{\sum_{j=1}^{k+1} \alpha_{i_{j}} g\left(x_{i_{j}}\right)}{\sum_{j=1}^{k+1} \alpha_{i_{j}}}\right) d x_{i_{1}} \cdots d x_{i_{k+1}} \\
\leq & \frac{\bar{w}^{n-k}}{\left(\begin{array}{c}
n-1 \\
k-1
\end{array}\right)} \sum_{1 \leq i_{1}<\cdots<i_{k} \leq n} \sum_{r=1}^{k} \alpha_{i_{r}} \int_{a}^{b} \cdots \int_{a}^{b}\left[\prod_{s=1}^{k} w\left(x_{i_{s}}\right)\right] f\left(\frac{\sum_{j=1}^{k} \alpha_{i_{j}} g\left(x_{i_{j}}\right)}{\sum_{j=1}^{k} \alpha_{i_{j}}}\right) d x_{i_{1}} \cdots d x_{i_{k}},
\end{aligned}
$$

that is,

$$
F_{k+1, n} \leq F_{k, n},
$$

which provides the remaining inequalities. The final equality is trivial.

Denote by

$$
\bar{F}_{k, n}=\frac{1}{\left(\begin{array}{c}
n-1 \\
k-1
\end{array}\right)(b-a)^{k}} \sum_{1 \leq i_{1}<\cdots<i_{k} \leq n} \sum_{r=1}^{k} \alpha_{i_{r}} \int_{a}^{b} \cdots \int_{a}^{b} f\left(\frac{\sum_{j=1}^{k} \alpha_{i_{j}} x_{i_{j}}}{\sum_{j=1}^{k} \alpha_{i_{j}}}\right) d x_{i_{1}} \cdots d x_{i_{k}}
$$

the value of $F_{k, n}$ obtained in the special case $w(x):=1$ and $g(x):=x$. In particular we have

$$
\bar{F}_{n, n}=\frac{1}{(b-a)^{n}} \int_{a}^{b} \cdots \int_{a}^{b} f\left(\sum_{i=1}^{n} \alpha_{i} x_{i}\right) d x_{1} \cdots d x_{n} .
$$

Then we have the following. 
Corollary 1. Let $f:[a, b] \rightarrow \mathbf{R}$ be convex and $\alpha_{i} \in(0,1)(i=1, \ldots, n)$ with $\sum_{i=1}^{n} \alpha_{i}=1$. Then

$$
f\left(\frac{a+b}{2}\right) \leq \bar{F}_{n, n} \leq \cdots \leq \bar{F}_{k+1, n} \leq \bar{F}_{k, n} \leq \cdots \leq \bar{F}_{1, n}=\frac{1}{b-a} \int_{a}^{b} f(x) d x .
$$

Remark 1. Corollary 1 provides a refinement of Theorem A, which may be written

$$
f\left(\frac{a+b}{2}\right) \leq \bar{F}_{n, n} \leq \bar{F}_{n-1, n} \leq \bar{F}_{1, n}
$$

We now establish some associated results.

Theorem 2. Let the assumptions of Theorem 1 be fulfilled. Then

$$
F_{k, n} \leq G_{k, n}:=\frac{(n-k) F_{1, n}+(k-1) F_{n, n}}{n-1} .
$$

Proof. Let $f_{k, n}$ be defined as in the proof of Theorem 1. It is known (see [1, p.173]) that

$$
f_{k, n}\left(x_{1}, \ldots, x_{n}\right) \leq \frac{(n-k) f_{1, n}\left(x_{1}, \ldots, x_{n}\right)+(k-1) f_{n, n}\left(x_{1}, \ldots, x_{n}\right)}{n-1}
$$

holds for all $k=1, \ldots, n$ and all real $n$-tuples $\left(x_{1}, \ldots, x_{n}\right)$. As in the previous theorem we may replace $x_{i}$ by $g\left(x_{i}\right)$. Multiplication by $\prod_{\ell=1}^{n} w\left(x_{\ell}\right)$ and integrating with respect to $x_{1}, x_{2}, \ldots, x_{n}$ yields the desired result.

Proposition 1. Under the assumptions of Theorem 1,

$$
F_{n, n}=G_{n, n} \leq F_{n-1, n} \leq G_{n-1, n} \leq \cdots \leq G_{k+1, n} \leq G_{k, n} \leq \cdots \leq G_{1, n}=F_{1, n} .
$$

Proof. By definition $F_{n, n}=G_{n, n}$ and $F_{1, n}=G_{1, n}$ and since $F_{n, n} \leq F_{1, n}$, we have $G_{k+1, n} \leq G_{k, n}$ for $1 \leq k<n$.

Corollary 2. Suppose the assumptions of Corollary 1 hold and that $\bar{G}_{k, n}$ is the value of $G_{k, n}$ when $w(x):=1$ and $g(x):=x$. Then

$$
\bar{F}_{n-1, n} \leq \bar{G}_{n-1, n} \leq \cdots \leq \bar{G}_{k+1, n} \leq \bar{G}_{k, n} \leq \cdots \leq \bar{G}_{1, n}=\bar{F}_{1, n} .
$$

Remark 2. Corollary 2 supplies an interpolation of the last inequality in (1).

\section{Convex Function Construction}

In this section we proceed to the construction of a convex, increasing function between the two sides of the first inequality in (2). 
Theorem 3. Let the assumptions of Theorem 1 be fulfilled and suppose the function $H_{f}:[0,1] \rightarrow \mathbf{R}$ is defined by

$H_{f}(t)=\frac{1}{\bar{w}^{n}} \int_{a}^{b} \cdots \int_{a}^{b}\left[\prod_{j=1}^{n} w\left(x_{j}\right)\right] f\left(t \sum_{i=1}^{n} \alpha_{i} g\left(x_{i}\right)+\frac{1-t}{\bar{w}} \int_{a}^{b} w(x) g(x) d x\right) d x_{1} \cdots d x_{n}$

Then

(i) $H_{f}$ is convex on $[0,1]$,

(ii)

$$
\begin{aligned}
& \min _{t \in[0,1]} H_{f}(t)=H_{f}(0)=f\left(\frac{1}{\bar{w}} \int_{a}^{b} w(x) g(x) d x\right) \\
& \max _{t \in[0,1]} H_{f}(t)=H_{f}(1)=\frac{1}{\bar{w}^{n}} \int_{a}^{b} \cdots \int_{a}^{b} f\left[\prod_{j=1}^{n} w\left(x_{j}\right)\right] f\left(\sum_{i=1}^{n} \alpha_{i} g\left(x_{i}\right)\right) d x_{1} \cdots d x_{n},
\end{aligned}
$$

(iii) $H_{f}$ is increasing on $[0,1]$.

Proof. (i) The function

$$
\phi(t):=t \sum_{i=1}^{n} \alpha_{i} g\left(x_{i}\right)+\frac{1-t}{\bar{w}} \int_{a}^{b} w(x) g(x) d x
$$

is linear for $t \in[0,1]$, so that $f \circ \phi$ is convex on $[0,1]$. Hence $H_{f}$ is convex on $[0,1]$.

(ii) From the convexity of $f$,

$$
H_{f}(t) \leq t H_{f}(1)+(1-t) H_{f}(0) .
$$

Also, by the first inequality in (2),

$$
H_{f}(0)=f\left(\frac{1}{\bar{w}} \int_{a}^{b} w(x) g(x) d x\right) \leq H_{f}(1) .
$$

Hence $H_{f}(t) \leq H_{f}(1)$.

Set $e(x):=x$. By the convexity of $f$,

$$
\begin{aligned}
H_{f}(t) & \geq f\left(H_{e}(t)\right)=f\left(\frac{t}{\bar{w}} \int_{a}^{b} w(x) g(x) d x+\frac{1-t}{\bar{w}} \int_{a}^{b} w(x) g(x) d x\right) \\
& =f\left(\frac{1}{\bar{w}} \int_{a}^{b} w(t) g(t) d t\right)=H_{f}(0) .
\end{aligned}
$$

(iii) Using the convexity of $H_{f}$ and $H_{f}(t) \geq H_{f}(0)$,

$$
\frac{H_{f}(u)-H_{f}(t)}{u-t} \geq \frac{H_{f}(t)-H_{f}(0)}{t} \geq 0
$$


for $0 \leq t<u \leq 1$. Hence $H_{f}(t) \leq H_{f}(u)$ for $t<u$.

\section{Second Refinement}

Theorem 4. Suppose $I$ is a real interval. Let $f: I \rightarrow \mathbf{R}$ be a convex function and let $h:[0,1] \rightarrow \mathbf{R}$ be defined by

$$
h(t)=\frac{1}{n} \sum_{i=1}^{n} f\left((1-t) x_{i}+t x_{i+1}\right),
$$

where $x_{i} \in I(1 \leq i \leq n)$ and $x_{n+1}=x_{1}$. Then $h$ is convex on $[0,1]$,

$$
\begin{gathered}
\min _{t \in[0,1]} h(t)=h(0)=f\left(\frac{\sum_{i=1}^{n} x_{i}}{n}\right), \\
\max _{t \in[0,1]} h(t)=h(1)=\frac{\sum_{i=1}^{n} f\left(x_{i}\right)}{n} .
\end{gathered}
$$

Proof. Let $t_{1}, t_{2} \in[0,1]$ and $a_{1}, a_{2}>0$ with $a_{1}+a_{2}=1$. Then

$$
\begin{aligned}
h\left(a_{1} t_{1}+a_{2} t_{2}\right) & =\frac{1}{n} \sum_{i=1}^{n} f\left(\left(1-\sum_{j=1}^{2} a_{j} t_{j}\right) x_{i}+\sum_{j=1}^{2} a_{j} t_{j} x_{i+1}\right) \\
& =\frac{1}{n} \sum_{i=1}^{n} f\left(\sum_{j=1}^{2} a_{j}\left[\left(1-t_{j}\right) x_{i}+t_{j} x_{i+1}\right]\right) \\
& \leq \frac{1}{n} \sum_{i=1}^{n} \sum_{j=1}^{2} a_{j} f\left(\left(1-t_{j}\right) x_{i}+t_{j} x_{i+1}\right) \\
& =a_{1} h\left(t_{1}\right)+a_{2} h\left(t_{2}\right),
\end{aligned}
$$

so $h$ is convex on $[0,1]$.

Consequently

$$
f\left(\frac{\sum_{i=1}^{n} x_{i}}{n}\right) \leq h(t) \leq \frac{\sum_{i=1}^{n} f\left(x_{i}\right)}{n},
$$

for all $x_{i} \in I(i=1, \ldots, n)$ and $t \in[0,1]$, whence (3) and (4) follow.

The special case $n=3$ was shown by Yang and $\mathrm{Wu}$ [4] and used to establish [4, Theorem 2.2]. The present result can be used to generalize, [4, Theorem 2.2] in a natural way. 


\section{Acknowledgement}

This research was done during a visit to the University of Adelaide by the first-named author and was supported by an ARC grant. The authors wish to thank the referee for useful suggestions.

\section{References}

[1] J. Pečarić, F. Proschan, Y. L. Tong, Convex Functions, Partial Orderings and Statistical Applications, Academic Press, New York, 1992.

[2] J. Pečarić, Remarks on an inequality of S. Gabler, J. Math. Anal. Appl., 184(1994), 19-21.

[3] G. -S. Yang and C. -S. Wang, Some refinements of Hadamard's inequality, Tamkang J. Math., 28(1997), 87-92.

[4] G. -S. Yang and H. -L. Wu, A refinement of Hadamard's inequality for isotonic linear functionals, Tamkang J. Math., 27(1996), 327-336.

Faculty of Electrical Engineering \& Computing, University of Zagreb, Unska 3, Zagreb, Croatia. ilko.brnetic@fer.hr

Applied Mathematics Department, The University of Adelaide, Adelaide SA 5005, Australia. cpearce@maths.adelaide.edu.au jpecaric@maths.adelaide.edu.au 